Original Research Paper

\title{
Automatic Counting for Live and Dead Cells from Trypan Blue-Stained Images by Image Analysis Based on Adaptive K- Means Clustering
}

\author{
${ }^{1}$ Su Mon Aung, ${ }^{2}$ Kanyanatt Kanokwiroon, ${ }^{1}$ Tonghathai Phairatana and ${ }^{1}$ Surapong Chatpun \\ ${ }^{1}$ Institute of Biomedical Engineering, Faculty of Medicine, Prince of Songkla University, Hat Yai, Songkhla 90110, Thailand \\ ${ }^{2}$ Department of Biomedical Sciences, Faculty of Medicine, Prince of Songkla University, Hat Yai, Songkhla 90110, Thailand
}

\author{
Article history \\ Received: 12-10-2018 \\ Revised: 8-01-2019 \\ Accepted: 26-02-2019 \\ Corresponding Author: \\ Surapong Chatpun \\ Institute of Biomedical \\ Engineering, Faculty of \\ Medicine, Prince of Songkla \\ University, Hat Yai, \\ Songkhla, Thailand \\ Email: surapong.c@psu.ac.th
}

\begin{abstract}
Computer-assisted image analysis can be employed to reduce the time consumed in the routine task such as cell counting. This study aimed to establish a method to perform this routine task based on an image analysis to automatically count live and dead cells after staining with trypan blue dye. Gray scale conversion and morphological operation were applied to the input images to enhance the image quality before image segmentation, then adaptive k-means clustering was applied to classify the groups of live and dead cells. Circular Hough transform and object labelling were carried out to identify the number of each cell type. The counting results from the proposed method were compared with the counting of three experts and the ImageJ software. The results showed that the proposed method had very high correlation with the results of the three experts in counting live cells $\left(\mathrm{R}^{2}>0.95\right)$ and was better than the counting results achieved by ImageJ. The number of dead cells counted by our program was in good agreement with the experts' counting $\left(\mathrm{R}^{2}>0.64\right)$. In conclusion, this study suggests that using new image analysis program can be confidently substituted for a manual counting in routine cell counting.
\end{abstract}

Keywords: Cell Counting, Adaptive K-Means Clustering, Morphological Reconstruction, Hough Transform, Trypan Blue

\section{Introduction}

Computer-assisted image analysis has been introduced in recent years in medical and biological research to make image-related work easier in both qualitative and quantitative ways (Merino et al.,2018; Kharghanian and Ahmadyfard, 2012; Poostchi et al., 2018). Cell counting is one of the routine tasks which is time-consuming and laborious. The accuracy of counting using conventional methods such as the standard Neubauer, Burker and Fuchs-Rosenthal chambers depends on the skill and experience of the professional or specialist who carries out the cell counting in the laboratory. A reliable image analysis method for counting cells would be advantageous and useful. Many algorithms have been developed for image analysis based on general and specific cell types.

The image segmentation technique has been devised to extract cell-sized objects by separating the boundaries of the objects from the background (Wang et al., 2015; Ambühl et al., 2012). The effectiveness of cell segmentation relies on the contrast between the cells and the background and the quality of the image. There are many segmentation methods currently being used, such as segmentation by histogram thresholding, Otsu thresholding, global thresholding, the Hough transform and watershed transform algorithms and kmeans clustering. The circular Hough transform is a frequently used method for detecting circular objects in an image (Rizon et al., 2005; Meng et al., 2018). However, it often suffers from degradation in performance, especially in terms of speed, because of the large number of edges created by a complex background or texture. In the analysis of white blood cells, some of the current techniques used are gradient vector flow, the snake algorithm and Zack thresholding which can be used for segmenting the nuclei of cells (Sahastrabuddhe, 2016).

Image post-processing includes feature extraction and morphological operations to identify objects and include erosion, dilation and morphological filtering. An opening operation is used to smoothen an image while a closing 
operation fills gaps and holes of an image (Sandor and Leahy, 1997). Feature extraction identifies features that contain quantitative information about objects of interest. Shape features frequently used in biological images are geometric parameters like cell area, cell perimeter and the ratio of the nucleus to the overall cell area, the boundary of the nucleus and the circularity index (Sahastrabuddhe, 2016). This image post-processing provides more specific data and enhances efficiency in cell detection.

As mentioned, the quality of the digital image and the types of cells in the image are factors to be considered for image analysis and cell counting. Images of trypan blue-stained cells viewed through a microscope are usually counted and calculated for the densities of live and dead cells to assess the effectiveness of novel cancer drug treatments. However, the quality of images for trypan blue-stained cells varies depending on the setup of the microscope, the light source, the quality of the stained images and the experience of the personnel performing the related tasks. These factors consequently effect on the counting accuracy and the image processing techniques can help to improve the counting accuracy. Therefore, we felt that a combination of suitable preprocessing, segmentation and post-processing techniques could enhance the efficiency of cell detection and cell counting for trypan blue-stained images. The aim of this work was to develop an algorithm to automatically count trypan blue-stained cells from light microscope images and to reliably distinguish between live and dead cells.

\section{Methodology}

Figure 1 shows the block diagram of the proposed method to count the trypan blue -stained cells from a light microscope image. The input image first underwent gray scale conversion and morphological reconstruction in the pre-processing step and then cell classification was conducted by the adaptive k-means clustering. Live and dead cells were counted after the post-processing step which employed a circular Hough transform and object labelling.

\section{Image Acquisition}

The data set of trypan blue stained-images was adopted from the cytotoxicity test of human breast cancer cell line. In brief, the human breast cancer cell line (MDA-MB-231) was treated with an anticancer drug and trypan blue dye, exposed to trypsin/EDTA and then $20 \mu \mathrm{L}$ of the mixture was pipetted into a hemocytometer. The trypan blue stained images were captured using an inverted Olympus IX51 light microscope equipped with an Olympus DP72 digital camera with a 2/3 inch CCD. The images were obtained in a bright field using Olympus UPLanFL N $10 \times 0.13$ as a standard objective lens. An example of digital input image of trypan blue stained cells was shown in Fig. 2. The image was cropped to a 16-square grid.

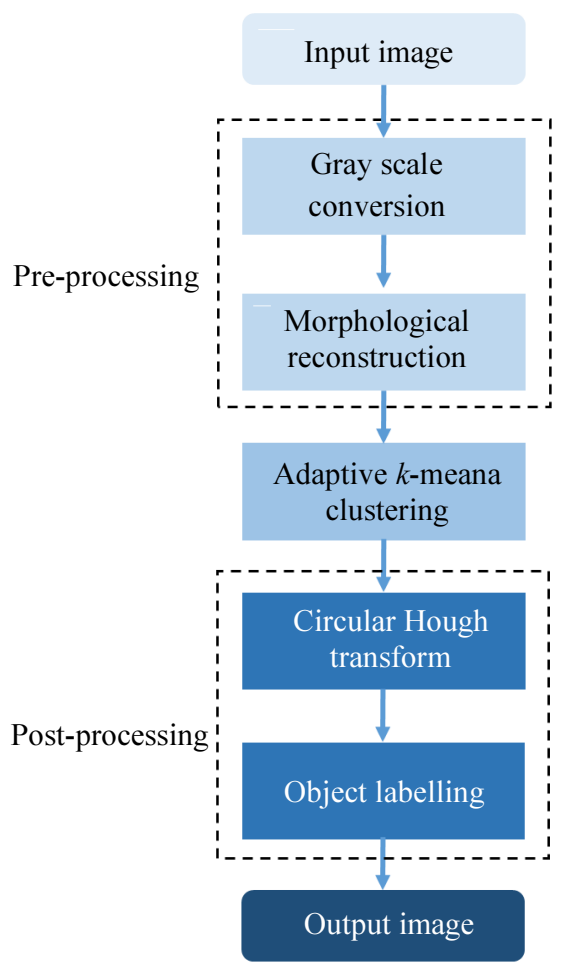

Fig. 1: Block diagram of the proposed method for trypan blue stained image 

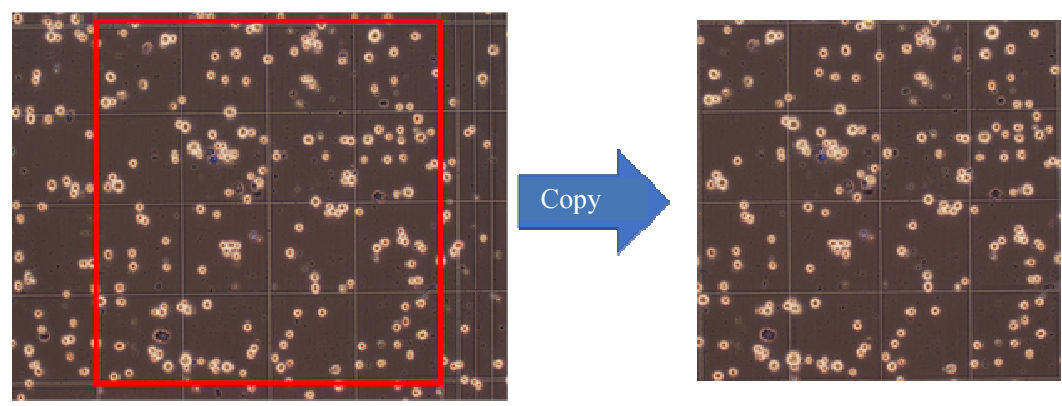

Fig. 2: Example of input image of trypan blue stained cells and cropped image for 16-square grid

\section{Pre-Processing}

Pre-processing of the images was conducted by converting the RGB image into a gray scale image. Conversion to gray scale image is necessary in order to handle the subsequent processes easier. RGB values are converted to grayscale values by forming a weighted sum of R, G and B components as in Equation (1):

$$
f_{\text {gray }}(\text { grayimage })=0.29 R+0.59 G+0.11 B
$$

where, $R, G$ and $B$ are the red, green and blue components measured in pixels, respectively.

Morphological concepts and methods have been proposed as constituting a powerful set of tools for extracting features from an image. Morphological techniques can be applied for image segmentation and can play a significant role in an algorithm for image information. Morphological opening, closing and erosion can be used to eliminate small objects and the consequent dilation tends to reconstruct the pattern of the objects that remain. These four operators can perform only on gray image or binary image with the following equations.

Erosion of a digital $\mathrm{f}$ with a structuring element $\mathrm{S}$ :

$$
(\varepsilon(f))(x)=\min _{s \in S} f(x+s)
$$

Dilation of a $\operatorname{digital} f$ with a structuring element $S$ :

$$
(\delta(f))(x)=\max _{s \in S} f(x+s)
$$

$$
\text { Opening: } \gamma(f)=\delta(\varepsilon(f))
$$

$$
\text { Closing: } \phi(f)=\varepsilon(\delta(f))
$$

In this study, image extraction was performed from a stained image including both live and dead cells. Both the morphological opening operation and the reconstruction of the opening pixels by erosion were performed together at the beginning by applying a disk structuring element that could be any kind of shapes. For this study, the 'disk' shape structuring element was chosen because staining cells were assumed a circular shape and preserved the nature of circular object without losing pixel information of original image. Structuring element was set to pixels (radius $=15$ ). However, the efficiency of this reconstruction depended on the shapes of cells and the radius of the structuring element.

Generally, opening eliminates bright details smaller than the structuring element, while closing suppresses dark features smaller than the structuring element. Opening can be also used to compensate for non-uniform background illumination. By subtracting this background from the original image, it was possible to form an image of the live and dead cells with a reasonably smooth background.

\section{Cell Classification Using Adaptive $k$-Means Clustering}

In general, image classification is based on the identification of the k-element in the data set that could be used to create an initial representation of clusters. In this study, the value of the k-element was set to 3 to group the objects in the images and three clusters were identified, namely: (1) dead cells, (2) total cells and (3) live cells. These clusters were based on the gray scale intensity of each object. The difference between the gray scale intensity of two pixels is measured with Euclidean distance. The k-element was formed into clustering seeds and the remaining clustering seeds of the data set were assigned to one of the clusters. The live and dead cells in the stained image were extracted using the adaptive kmeans clustering algorithm. In this clustering algorithm, the Euclidean distance of two elements $E_{1}=\left\{E_{11}\right.$, $\left.E_{12}, \ldots, E_{1 n}\right\}$ and $E_{2}=\left\{E_{21}, E_{22}, \ldots, E_{2 n}\right\}$ is given by $\sqrt{\left(E_{11}-E_{21}\right)^{2}+\left(E_{12}-E_{22}\right)^{2}+\ldots+\left(E_{1 n}-E_{2 n}\right)^{2}}$. The distance between any two clusters is minimized as well as the two closest clusters identified. In this study, cluster-1 and cluster-3, live and dead cells, were chosen to be counted.

\section{Post-Processing}

The segmented image of the live cells (cluster-3) from the adaptive k-means was used as the input to the circular Hough transform process. The circular Hough 
transform has been identified as a particularly robust tool to detect the parametric curves in images. It achieves a voting process that matches image edge points into manifolds in an approximately defined parameter range. The circular Hough transform was applied in this study to locate the circular patterns within an image and to perform the counting label. A circular pattern is described by Equation (6):

$\left(x_{p}-x_{0}\right)^{2}+\left(y_{p}-y_{0}\right)^{2}=r^{2}$

where, $x_{0}$ and $y_{0}$ are the coordinates of the center and $r$ is the radius of the circle.

Labelling object was used to count cells from trypan blue-stained images. The counting of dead cells was accomplished by identifying the number of connected components in the segmented image from the adaptive kmeans clustering (cluster-1) and then the connected objects were counted in each segmented image.

\section{Accuracy Verification}

In this study, the counting results of the newly developed technique were compared with the results from an ImageJ assessment and three experts who counted the different cells in the images using the standard technique. Thirty-six trypan blue stained images were assessed in this way to evaluate the accuracy of our proposed technique. The CellContingMacro2 v1-01 plugin of ImageJ was employed to count the different cells. The accuracy of the cell counting results was determined as shown in Equation (7):

$$
\% \text { Accuracy }=100-(\mid \text { Pr ogram }- \text { Expert } \mid / \text { Expert }) \times 100
$$

Linear correlation to compare the counting methods was performed to determine the relationship between the developed technique and the conventional method.

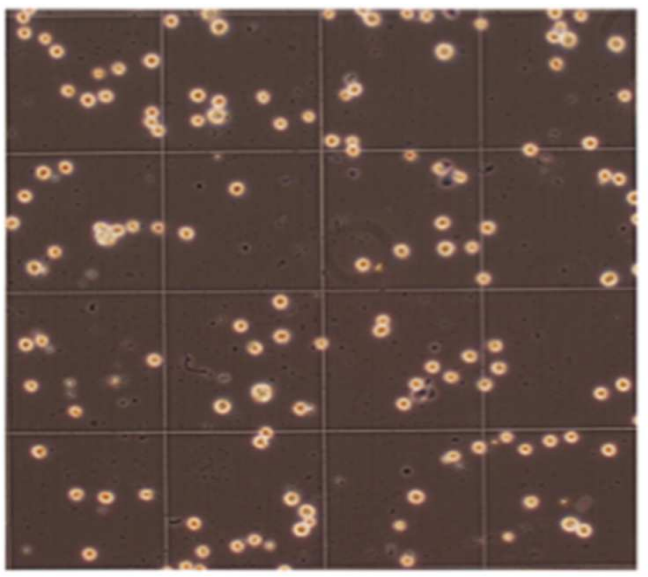

Original image (RGB image)

\section{Results and Discussion}

Thirty-six light-microscope trypan blue-stained images were obtained as RGB images and then they were converted to gray-scale images as shown in Fig. 3. The gray scale images were used as inputs to perform pre-processing image techniques with the opening and closing morphological operators. Then the reconstruction of the morphological operations was applied with a diskstructuring element and the live and dead cells were reconstructed perfectly as shown in Fig. 4. It can be noticed that the cells in the image after pre-processing have greater contrast with the background and brightness, similar to the previous studies (Hassanpour et al., 2015; Sreedhar et al., 2012). It was also found that the morphological operations structuring element was able to eliminate erroneous pixels in the image, although another study has found that if either larger or smaller pixels are used in images, then the segmentation quality might be affected (Liew et al., 2010).

Figure 5 illustrates the three clusters, dead cells, total cells and live cells, resulting from the adaptive k-means clustering. Some studies found that the adaptive k-means clustering required an accurate pre-processing process in order to obtain an accurate cell classification, but when this process was followed, the results were much better than standard k-means clustering (Moftah et al., 2014). Classification is improved through the application of adequate pre-processing steps such as gray-scale conversion and morphological operations to remove grid lines. In our study, using the circular Hough transform after segmentation from the adaptive k-means clustering, the live cells were detected and extracted from the dead cells and the background as shown in Fig. 6, in which it can be noted that the shape of the live cells is circular and they are also brighter than the dead cells, indicating that the circular Hough transform can detect live cells efficiently.

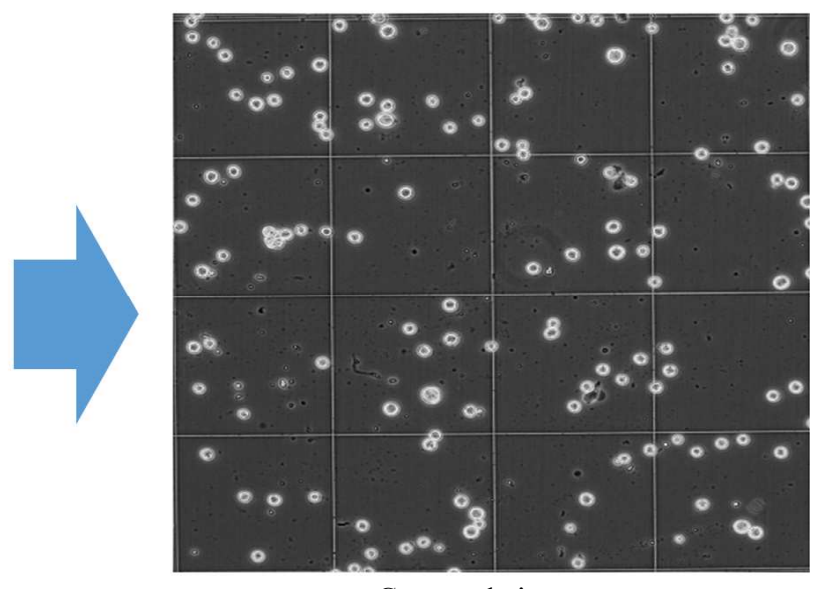

Gray scale image

Fig. 3: Original (RGB) to gray-scale converted image 


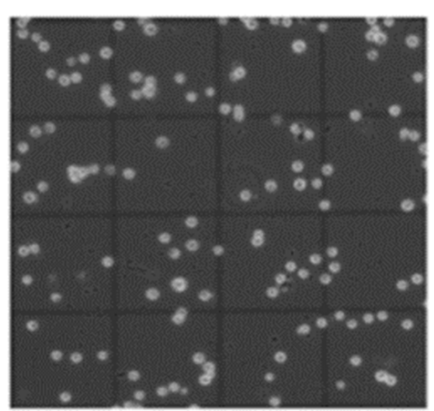

(a)

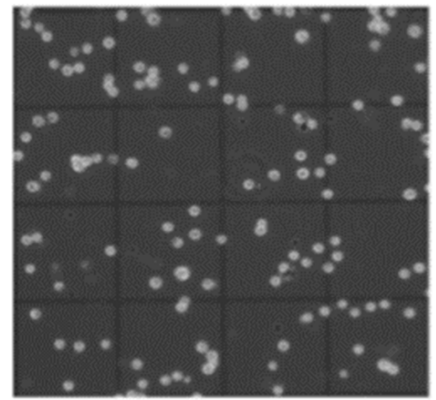

(c)

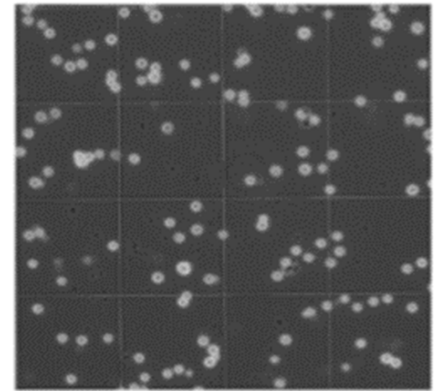

(b)

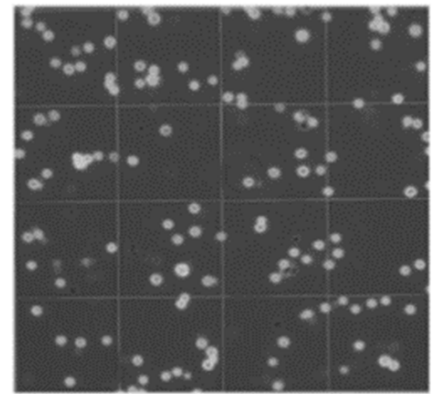

(d)

Fig. 4: Morphological operations: image after (a) Opening operation (b) opening by reconstruction (c) opening-closing operation and (d) opening-closing reconstruction

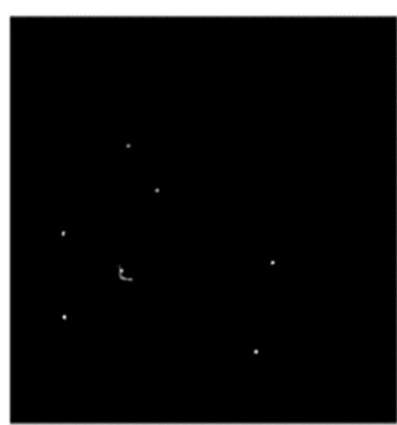

(a)

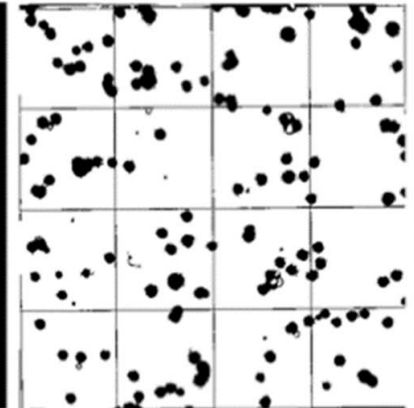

(b)

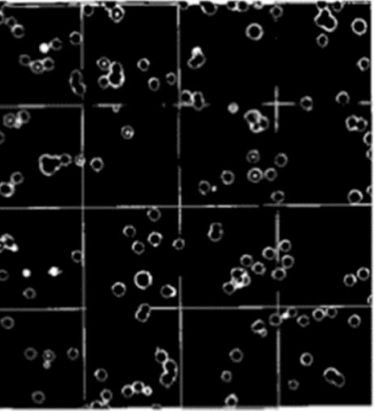

(c)

Fig. 5: Adaptive k-means clustering: (a) cluster-1 dead cells (b) cluster-2 total cells and (c) cluster-3 live cells

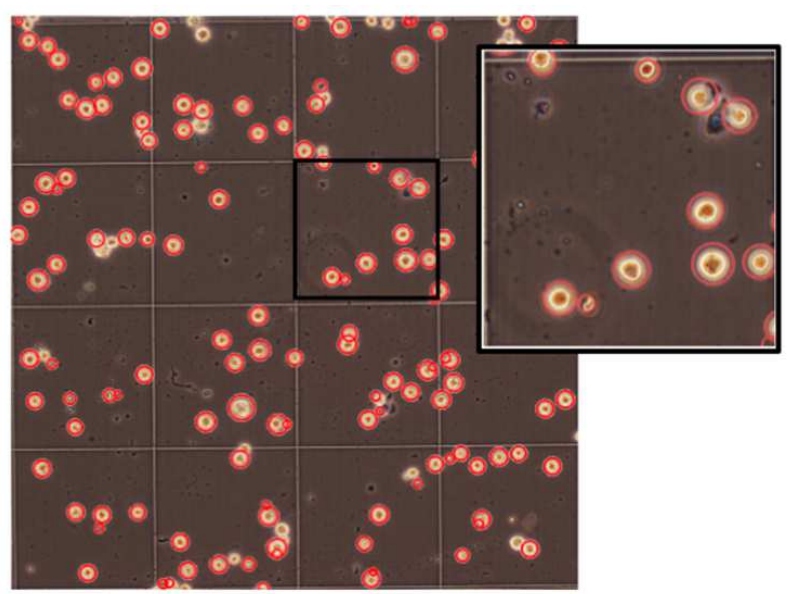

Fig. 6: Example of cluster-3 image after circular Hough transform for live cell detection 


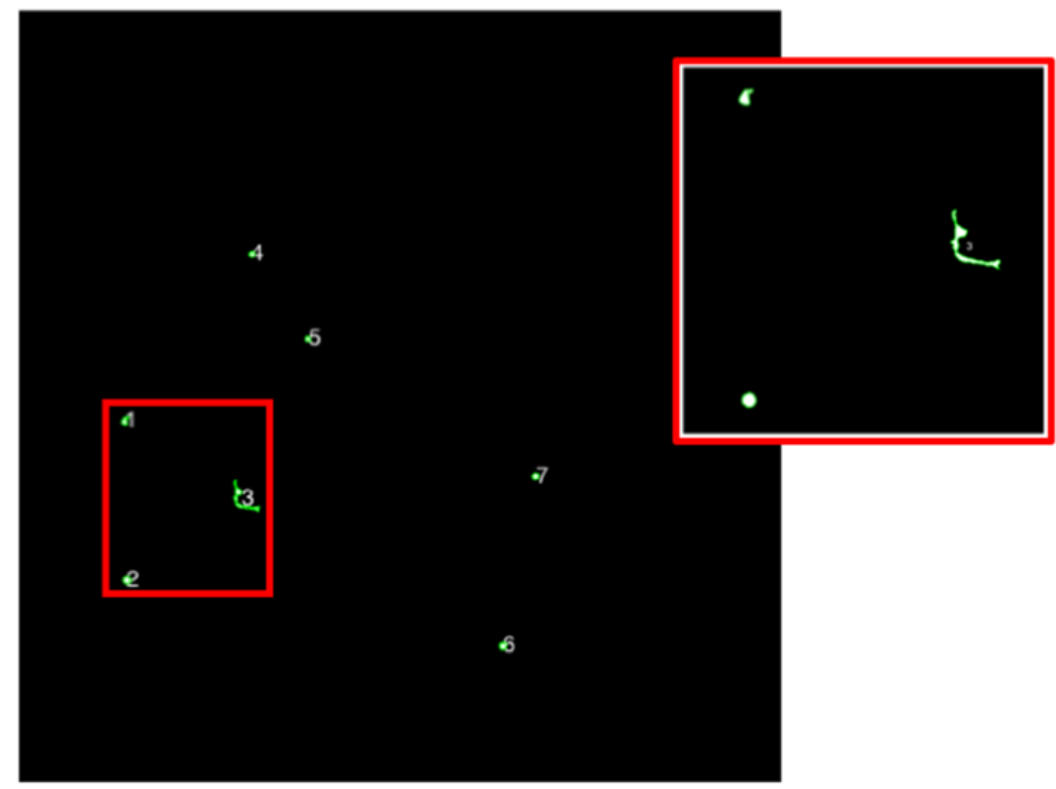

Fig. 7: Example of cluster-1 image after labeling for dead cell detection

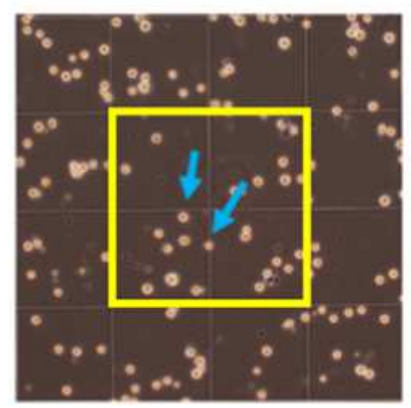

(a)

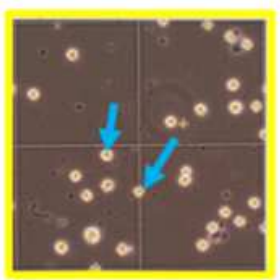

(b)

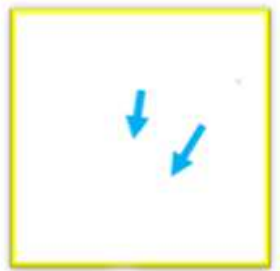

(c)

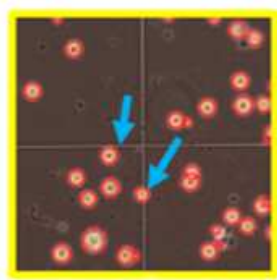

(d)

Fig. 8: Comparison of counting procedures: (a) original image (b) manual counting (c) ImageJ counting and (d) our method

In a study recently reported by Acharya and Kumar (2018), the circular Hough transform gave more accurate counting Results of Red Blood Cells (RBCs) than the labeling algorithm, which they believed was because of the circular shape of RBCs (Acharya and Kumar, 2018). The counting of the dead cells was accomplished by finding the number of connected components in the segmented image of the adaptive k-means clustering (cluster-1), as presented in Fig. 7. However, there remain many challenges in accurately identifying dead cells due to their irregular shape, variable sizes and poor contrast to the background.

In this study, the proposed method was compared with the open source program ImageJ and the manual counting by three experts. It was found that ImageJ could not distinguish live and dead cells accurately from the original image, as shown in Fig. 8. 


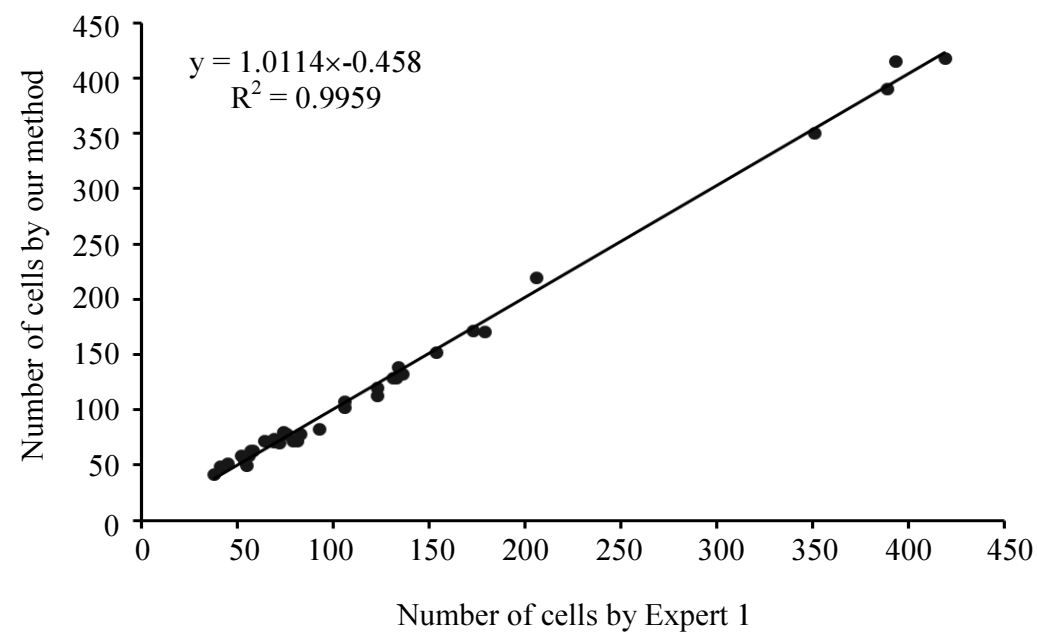

(a)

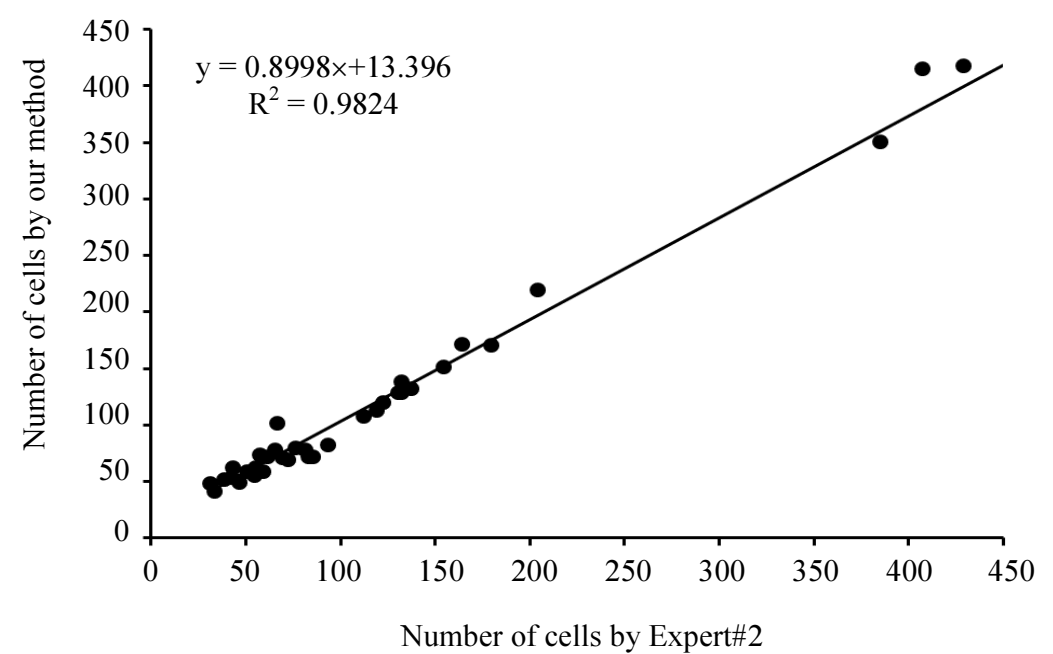

(b)

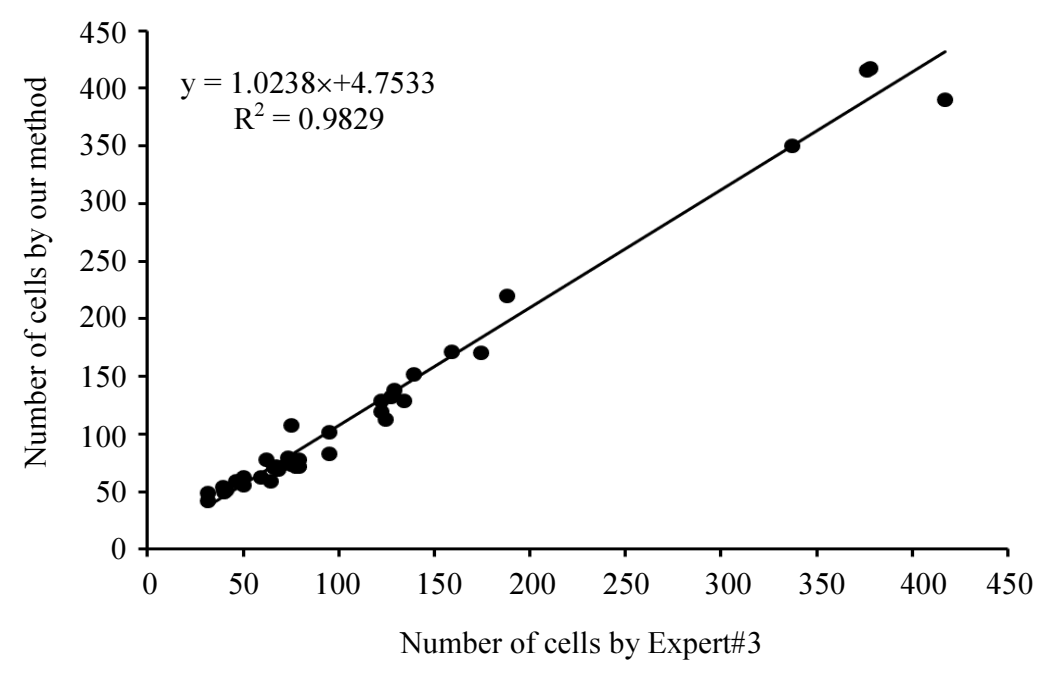

(c)

Fig. 9: Accuracy verification: (a)-(c) linear correlation of our method and three experts for live cell counting 


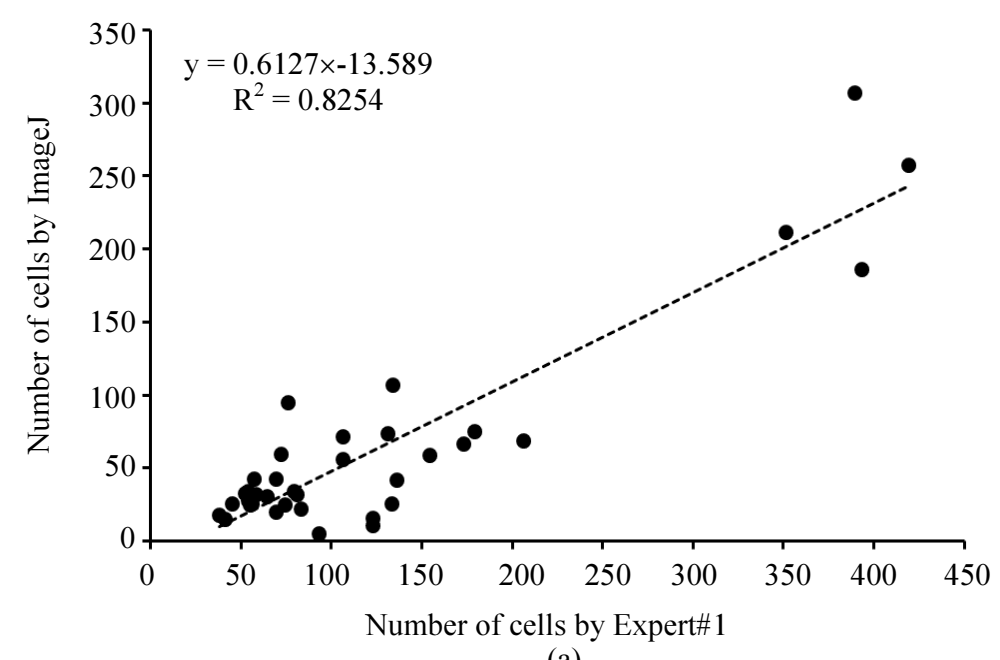

(a)

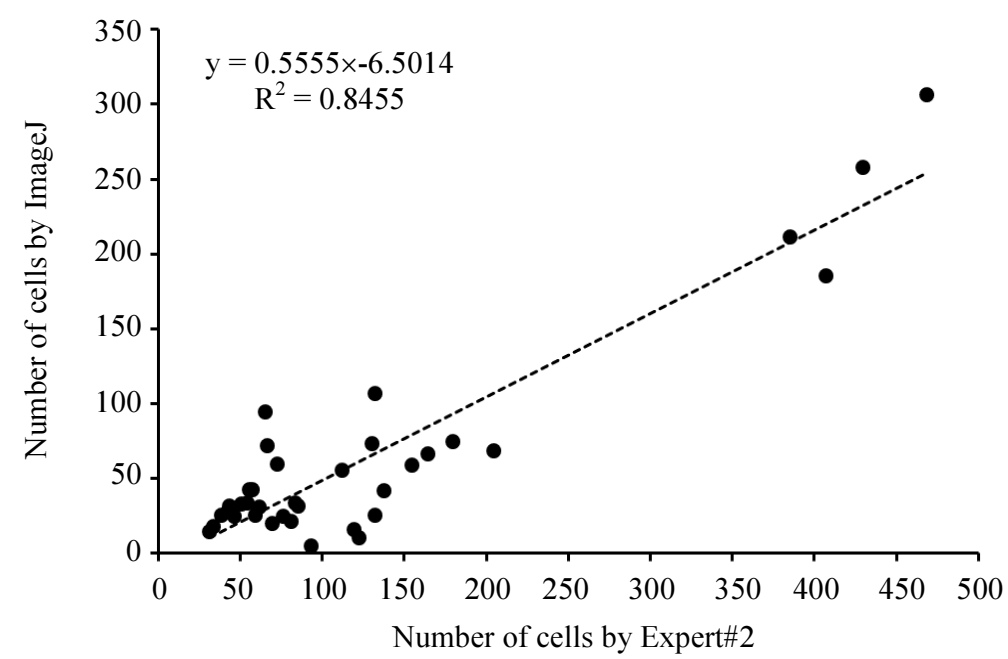

(b)

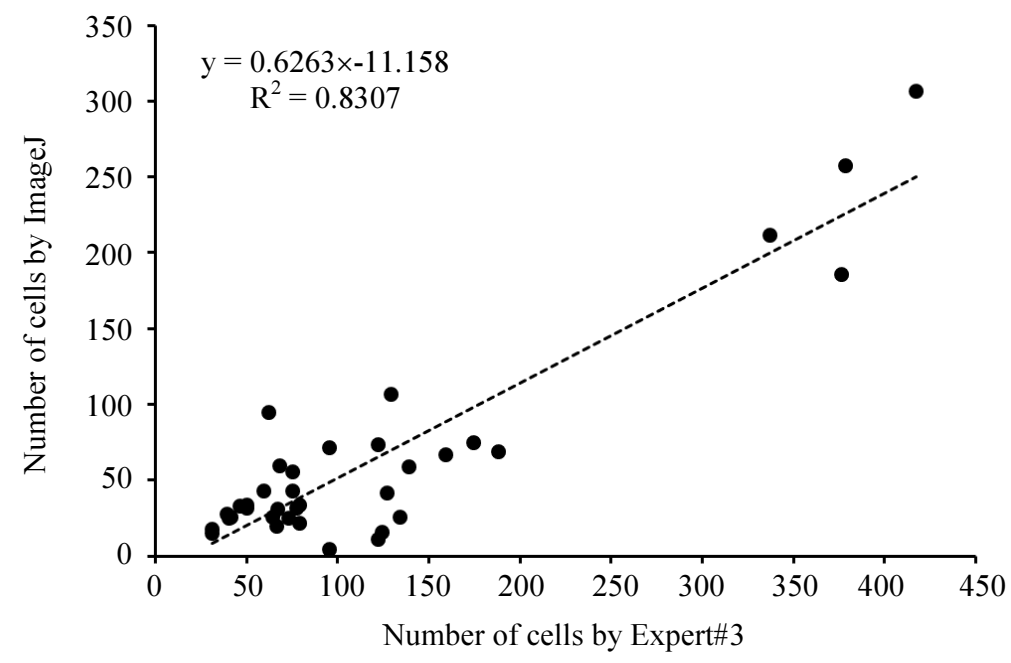

(c)

Fig. 10: Accuracy verification: (a)-(c) linear correlation of ImageJ and three experts for live cell counting 


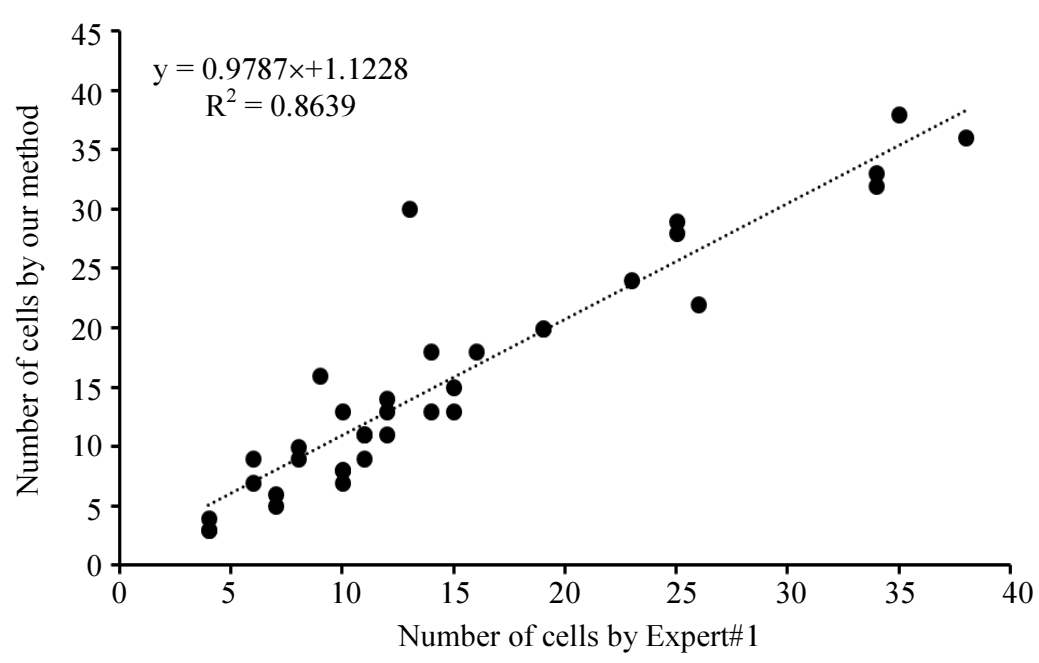

(a)

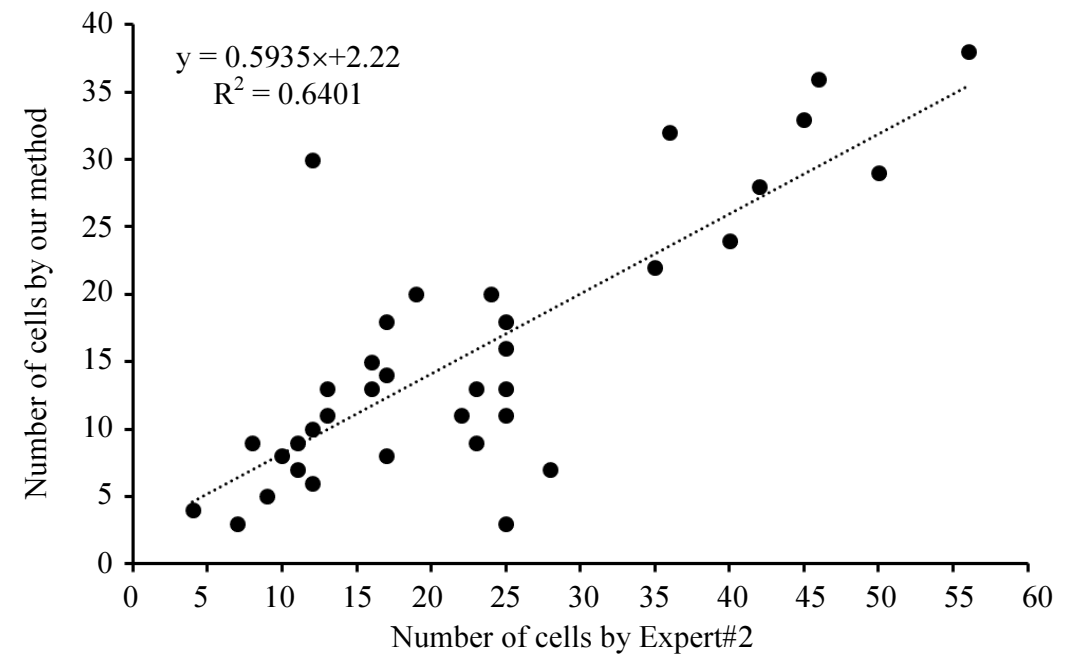

(b)

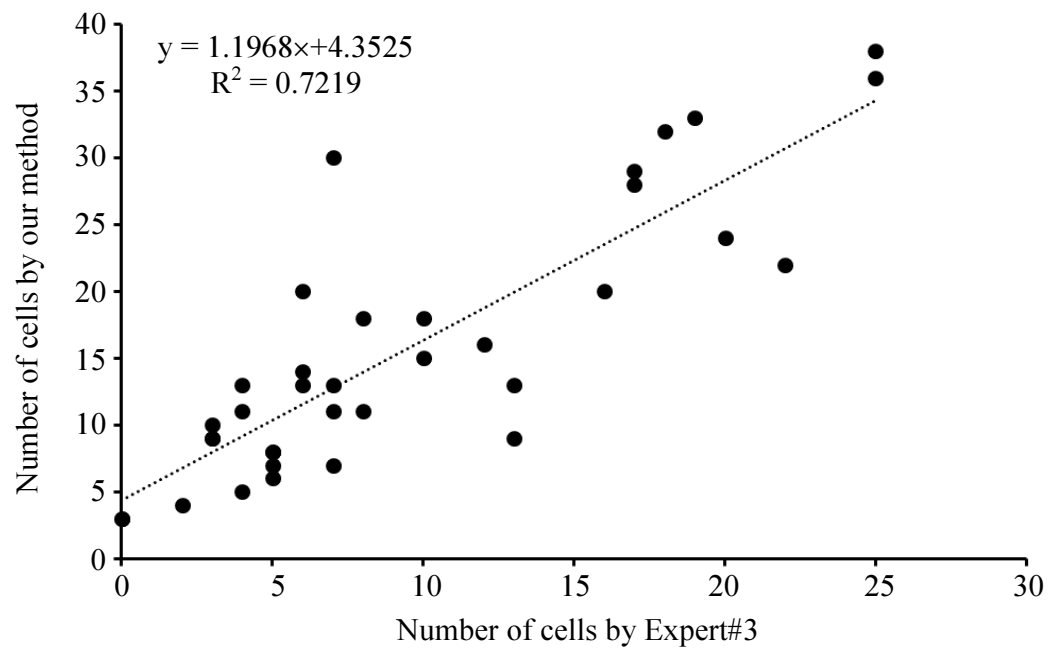

(c)

Fig. 11: Accuracy verification: (a)-(c) linear correlation of proposed method and three experts for dead cell counting 
The study found that the counting of live cells by the proposed method consistently fit a regression line with very high coefficient of determination $\left(R^{2}>0.95\right)$, when using the counts conducted by the three experts as the 'gold standard' as shown in Fig. 9. On the other hand, the live cell counting results of ImageJ had a lower coefficient of determination $\left(\mathrm{R}^{2}<0.85\right)$ when compared with the three experts, as shown in Fig. 10. Therefore, these results suggest that the counting of live cells from the proposed method can reasonably be accepted as as accurate as counting by experienced human workers. The average coefficient of determination for the results of counting dead cells between our method and the three experts was also quite good $\left(\mathrm{R}^{2}>0.64\right)$ as shown in Fig. 11, while ImageJ had much lower success rate to detect dead cells as the cell counter plug-in was not able to distinguish dead cells from live cells and the background in the trypan blue-stained cell images. However, our findings do not mean ImageJ is not suitable for cell counting, as other studies have reported on the effectiveness of ImageJ in cell counting (Grishagin, 2015; Choudhry, 2016). From this difference, we suggest that the counting accuracy of Image J depends on the cell types and cell preparation process prior to image acquisition.

The development of the image analysis assisted system for cell counting can provide more specific cell type comparisons, at less expensive cost, compared to currently available commercial counting software. Furthermore, our proposed method took $53 \mathrm{sec}$ per $4140 \times 3096$ pixels image on the average while the counting time from each expert varied from $36 \mathrm{sec}$ to 81 sec. To achieve higher counting reliability for routine trypan blue-stained cells counting tasks, there are several concerns related to the quality of input images, features of cell and pre- and post-processing techniques for further development (Putzu et al., 2014; Seyyedyazdi and Hassanpour, 2018). Moreover, a more user-friendly interface should be developed to facilitate the process.

\section{Conclusion}

We proposed a digital image processing method for the automatic cell counting of live and dead cells from light microscope-obtained trypan blue-stained cell images. Our results indicated that the proposed cell counting method is able to efficiently count the live and dead cells presented in a trypan blue-stained image with high correlation to the experts' counting. This proposed method removed hemocytometer grid lines which can cause difficulties in segmentation process. Adaptive kmeans clustering classified live and dead cells from a gray scale image as cluster-1 and cluster-3 respectively. At the final stage, a circular Hough transform and an object labelling were applied to detect and count live and dead cells. The counting results could be reported in a tabular format for later use. Furthermore, our proposed method is less time consumption, user-friendly and suitable for users who may not have a deep knowledge of image processing. Finally, to increase the level of accuracy, it is highly recommended to emphasize on image segmentation for the classification of the live and dead cells from the images.

\section{Acknowledgement}

Our technical discussions with Assoc. Prof. Pornchai Phukpattaranont from the Faculty of Engineering, Prince of Songkla University and Ms. Pamina Bernou, an internship student from Telecom Physique Strasbourg, France, were also very useful. We also thank the International Affairs Office of the Faculty of Medicine, Prince of Songkla University for editing the English of the manuscript.

\section{Funding Information}

The authors would like to acknowledge the Thailand's Education Hub for the Southern Region of ASEAN Countries (TEH-AC) scholarship given to Ms. Su Mon Aung from the Graduate School, Prince of Songkla University.

\section{Author's Contributions}

Su Mon Aung: Developed the algorithm for image processing, analyzed the results and prepared the manuscript.

Kanyanatt Kanokwiroon: Provided the images for this work, analyzed and discussed the results.

Tonghathai Phairatana: Analyzed the results and reviewed the manuscript.

Surapong Chatpun: Initiated the idea of the work, organized the research study, analyzed the results and wrote the manuscript.

\section{Ethics}

This article is original and contains unpublished material. The corresponding author confirms that all of the other authors have read and approved the manuscript and no ethical issues involved.

\section{References}

Acharya, V. and P. Kumar, 2018. Identification and red blood cell automated counting from blood smear images using computer-aided system. Medical Biological Engineer. Comput., 56: 483-489. DOI: $10.1007 / \mathrm{s} 11517-017-1708-9$

Ambühl, M.E., C. Brepsant, J.J. Meister, A.B. Verkhovsky and I.F. Sbalzarini, 2012. Highresolution cell outline segmentation and tracking from phase-contrast microscopy images. J. Microscopy, 245: 161-170. DOI: 10.1111/ijlh.12832 
Choudhry, P., 2016. High-throughput method for automated colony and cell counting by digital image analysis based on edge detection. PLoS One, 11: e0148469. DOI: 10.1371/journal.pone.0148469

Grishagin, I.V., 2015. Automatic cell counting with ImageJ. Analytical Biochemistry, 473: 63-65. DOI: 10.1016/j.ab.2014.12.007

Hassanpour, H., N. Samadiani and S.M. Mahdi Salehi, 2015. Using morphological transforms to enhance the contrast of medical images. Egyptian J. Radiol. Nuclear Medicine, 46: 481-489.

DOI: 10.1016/j.ejrnm.2015.01.004

Kharghanian, R. and A.R. Ahmadyfard, 2012. Extracting vessel centerlines from retinal images using topographical properties and directional filters. Int. J. Engineer. Transact. B: Applicat., 25: 315-324. DOI: 10.5829/idosi.ije.2012.25.04b.07

Liew, L.H., B.Y. Lee and M. Chan, 2010. Cell detection for bee comb images using circular Hough transformation. Proceedings of the International Conference on Science and Social Research, Dec. 57, IEEE Xplore Press, Malaysia, pp: 191-195. DOI: $10.1109 /$ CSSR.2010.5773764

Meng, Y., Z. Zhang, H. Yin and T. Ma, 2018. Automatic detection of particle size distribution by image analysis based on local adaptive canny edge detection and modified circular Hough transform. Micron, 106: 34-41. DOI: $10.1016 /$ j.micron.2017.12.002

Merino, A., L. Puigví, L. Boldú, S. Alférez and J. Rodellar, 2018. Optimizing morphology through blood cell image analysis. Int. J. Laboratory Hematol., 40: 54-61. DOI: 10.1111/ijlh.12832.

Moftah, H.M., A.T. Azar, E.T. Al-Shammari, N.I. Ghali and A.E. Hassanien et al., 2014. Adaptive k-means clustering algorithm for MR breast image segmentation. Neural Comput. Applicat., 24: 1917-1928. DOI: 10.1007/s00521-013-1437-4
Poostchi, M., K. Silamut, R.J. Maude, S. Jaeger and G. Thoma, 2018. Image analysis and machine learning for detecting malaria. Translational Res., 194: 36-55. DOI: 10.1016/j.trs1.2017.12.004

Putzu, L., G. Caocci and D.C. Ruberto, 2014. Leucocyte classification for leukaemia detection using image processing techniques. Artificial Intelligence Med., 62: 179-191. DOI: 10.1016/j.artmed.2014.09.002

Rizon, M., H. Yazid, P. Saad, A.Y. Md Shakaff and A.R. Saad et al., 2005. Object detection using circular Hough transform. Am. J. Applied Sci., 2: 1606-1609.

Sahastrabuddhe, A.P., 2016. Counting of RBC and WBC using image processing: A Review. Int. J. Res. Eng.. Technol., 5: 356-360.

Sandor, S. and R. Leahy, 1997. Surface-based labeling of cortical anatomy using a deformable atlas. IEEE Trans. Medical Imag., 16: 41-54.

DOI: $10.1109 / 42.552054$

Seyyedyazdi, S. and H. Hassanpour, 2018. Improving super-resolution techniques via employing blurriness information of the image. Int. J. Eng. Trans. B: Applic., 31: 241-249.

DOI: $10.5829 /$ ije.2018.31.02b.07

Sreedhar, K., 2012. Enhancement of images using morphological transformations. Int. J. Comput. Sci. Inform. Technol., 4: 33-50. DOI: $10.5121 /$ ijcsit.2012.4103

Wang, Y., Z. Zhang, H. Wang and S. Bi, 2015. Segmentation of the clustered cells with optimized boundary detection in negative phase contrast images. PLoS One, 10: e0130178.

DOI: 10.1371/journal.pone.0130178 